\title{
2. Effects of Stereotaxic Thalamotomy to Intractable Pain and Numbness
}

\author{
Tatsuyuki Kudo and Nobuo YosHII \\ Division of Neurological Surgery, Dept. of Surgery, \\ School of Med., Keio University \\ Shiro SHIMIzU \\ Surgery, Tokyo Musashino Hosp. \\ Sadao Aikawa and Hiroshi Nakahama \\ Seishin Igaku Institute
}

There have been many devices for the relief operation for intractable pain and the target points that pain was safely eliminated by intervening thalamus were Centré median, Nucl. parafascicularies and Lamina medularis.

We obtained the good effects in two cases by intervening different part of thalamus, where mentioned above, a part of Nucl. pulvinaris. Also report two cases which we were able to eliminate numbness with pain on one side of the body by applying the same procedure to patients with cerebro-vascular disturbance.

Their chief complaints were "Sever pain on the left side of the body," "Continuous and sever pain in the lower abdomen," "Abnormal sensation (numbness and pain) on the right side of the body," and "Abnormal sensation (numbness, pain and coldness) on the right side." In all cases pain and numbness vanished and until today no sign of relapse has been found ( 2 to 6 months duration).

The effect of stereotaxic operations are not established yet because of the occasional recurrence of the pain. In the reviewing literatures, recognition of the pain in the thalamus is not confirmed yet but Centre median is one of the most important one. Posterior group of the thalamus (substituted PO) seems to be an important site of the pain recognition. PO receives the projection of pain and touch sensation. Therefore it may be reasonable that the destruction of this part of the thalamus eliminates spontaneous pain sensation.

We intervened the part, which we thought Mountcastle's PO in human, 16-17 $\mathrm{mm}$ from the midline to lateral and 6-7 $\mathrm{mm}$ from medial wall of posterior horn. We should not draw conclusions from our observation hastilt, because of a short period of time after the operation and no autopsy with histological investigation. 